\title{
On the Direct and Radiated Components of the Collisional Particle Pressure in Liquid-Solid Flows *
}

\author{
R. ZENIT, M.L. HUNT and C.E. BRENNEN \\ Division of Engineering and Applied Science, California Institute of Technology, Mail Code 104-44, \\ Pasadena, CA 91125, U.S.A.
}

\begin{abstract}
In a recent study the collisional particle pressure was measured for liquid fluidized beds and liquid-solid flows. The particle pressure was defined as the 'additional pressure' generated by the presence of the particulate-solid phase in a liquid-solid mixture. The particle pressure generated by collisions of particles was found to be composed of two main contributions: one from pressure pulses generated by direct collisions of particles against the containing walls (direct component), and a second one from pressure pulses due to collisions between individual particles that are transmitted through the liquid (radiated component). This paper presents a summary of the technique to measure the particle pressure and the main results of that study.

Additional experiments were performed to further study each one of the components of the particle pressure. The direct component was studied by impacting particles on the active face of the pressure transducer. The magnitude of the measured impulse was found to be related to the impact velocity, the mass and the size of the impacting particle. By comparing the measurements with the predictions from Hertzian theory, a quantification of the interstitial fluid effects could be obtained. The radiated component was investigated by generating binary collisions of particles in the vicinity of the transducer. The magnitude of the measured impulse was found to be a function of fluid density, particle size and impact velocity. Predictions based on impulse-pressure theory were obtained and compared with the experimental measurements. The model results showed good agreement with the experimental measurements.
\end{abstract}

Key words: particle pressure, liquid-solid flow.

Abbreviations: PDF - Probability Density Function

\section{Introduction}

Interest in multiphase flows is motivated by many industrial applications as well as many natural processes. Flows involving solid-fluid mixtures occur in many chemical, petroleum and mining engineering applications. The lack of basic understanding of such mixtures represents an important limitation to better design of devices that handle liquid-solid flows. Even small improvements in the performance these devices could have an important economic impact.

* Dedicated to Leen van Wijngaarden, a true scholar, from whom C.E.B. learnt much about fluid mechanics. 
The nature of particulate two-phase flow is complex, and the basic governing equations for such systems are still a matter of current research. Models of dispersed two-phase flows are usually expressed in terms of a set of conservation equations for each of the phases. The momentum conservation equations are coupled through an interaction force term. Difficulties with this approach arise when dealing with the discrete nature of the solid phase. Pressures, $P_{f}$, on the dispersed phase and $P_{p}$ in the discrete phase, are defined and the corresponding pressure gradient terms are included in the momentum conservation equation. While $\partial P_{f} / \partial x_{i}$ does not impose any conceptual difficulty, the physical meaning of $\partial P_{p} / \partial x_{i}$ is less clear. The particle pressure, or granular pressure, results from the interactions between individual particles and solid boundaries. Whether it contains only particle-to-particle interactions or, in addition, includes indirect interactions through the interstitial fluid is a matter of debate. Many researchers have developed models for the particle pressure $[2,4,6,7]$, but none of these have been corroborated experimentally due to the difficulties encountered in the measurement of the particle pressure.

Recently, Zenit et al. [14] reported measurements of the collisional particle pressure at the walls of fluidized beds and liquid-solid flows using a flush mounted high-frequency-response pressure transducer to collect data on the collision of particles with the active face of the transducer. Two distinct contributions to the particle pressure were identified: a direct component, the result of direct particle collisions against the containing walls; and a radiated component, the result of pressure pulses between particles which are transmitted through the interstitial fluid. The present paper presents a more detailed analysis of these two contributions of the particle pressure.

\section{Particle Pressure Measurements}

In a recent paper Zenit et al. [14] measured the particle pressure for liquid-solid mixtures. Individual collisions of particles last for only few tens of microseconds, and hence a high-frequency pressure transducer is required. Zenit et al. used a piezoelectric dynamic pressure transducer, capable of responding to changes in pressure in less than 2 microseconds. Experiments were performed in the vertical section of a water loop shown in Figure 1. The pressure transducer was flushmounted to a side wall of the test section. A high-pass filter eliminated pressure fluctuations below $1 \mathrm{kHz}$. Thus, only collision-generated pressure pulses were registered. A data acquisition system was programmed to capture individual collision pressure pulses continuously. Vertical gravity driven flows of glass particles and liquid fluidized beds of steel, nylon and glass particles were studied (see Table I). A large number of individual pressure pulses were obtained to calculate the particle pressure. Two different test sections were used with 5.1 and $10.2 \mathrm{~cm}$ circular crosssection. The solid fraction, $v$, of the mixture was measured using an impedance volume fraction meter [9]. 


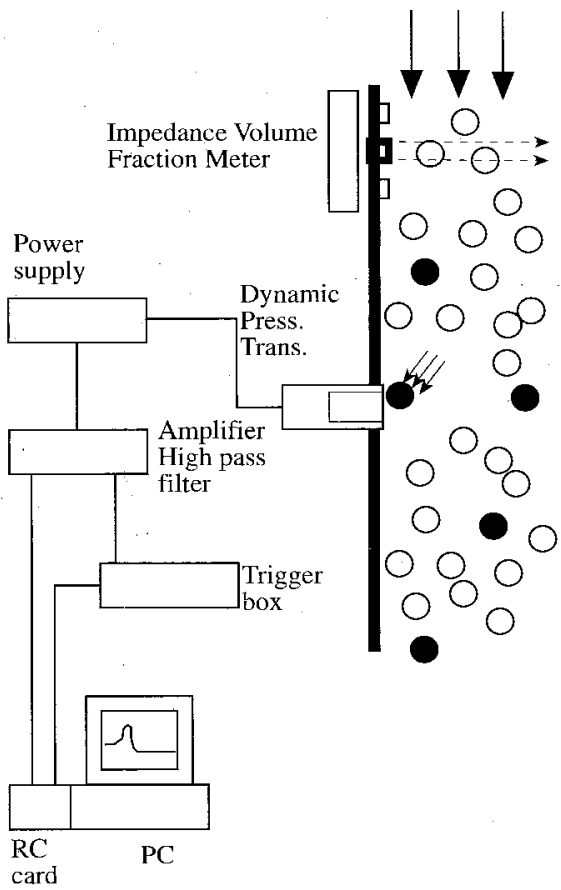

Figure 1. Experimental setup and instrumentation.

Table I. Properties of particles used in experiments.

\begin{tabular}{llllr}
\hline Material & $d_{p}[\mathrm{~mm}]$ & $\rho_{p} / \rho_{f}$ & $u_{t}[\mathrm{~cm} / \mathrm{s}]$ & $\mathrm{Re}_{t}$ \\
\hline Glass + & 2.06 & 2.54 & 22.7 & 452 \\
Glass $\times$ & 3.00 & 2.54 & 31.8 & 954 \\
Glass * & 3.96 & 2.54 & 36.8 & 1338 \\
Glass $\odot$ & 6.00 & 2.54 & 47.4 & 2583 \\
Steel $\oplus$ & 4.50 & 7.78 & 89.6 & 3665 \\
Nylon $\otimes$ & 6.35 & 1.14 & 13.6 & 785 \\
PVC $\odot$ & $3.41^{a}$ & 1.43 & 29.7 & 440 \\
\hline
\end{tabular}

${ }^{a}$ Equivalent diameter of the cylindrical shape.

The time average particle pressure was calculated as

$$
P_{p}=\frac{1}{T} \sum_{i=1}^{n} I_{i},
$$

where $T$ is the total time to capture $n$ pressure pulses, each with an impulse, $I$,

$$
I=\int_{0}^{\tau} P(t) \mathrm{d} t,
$$




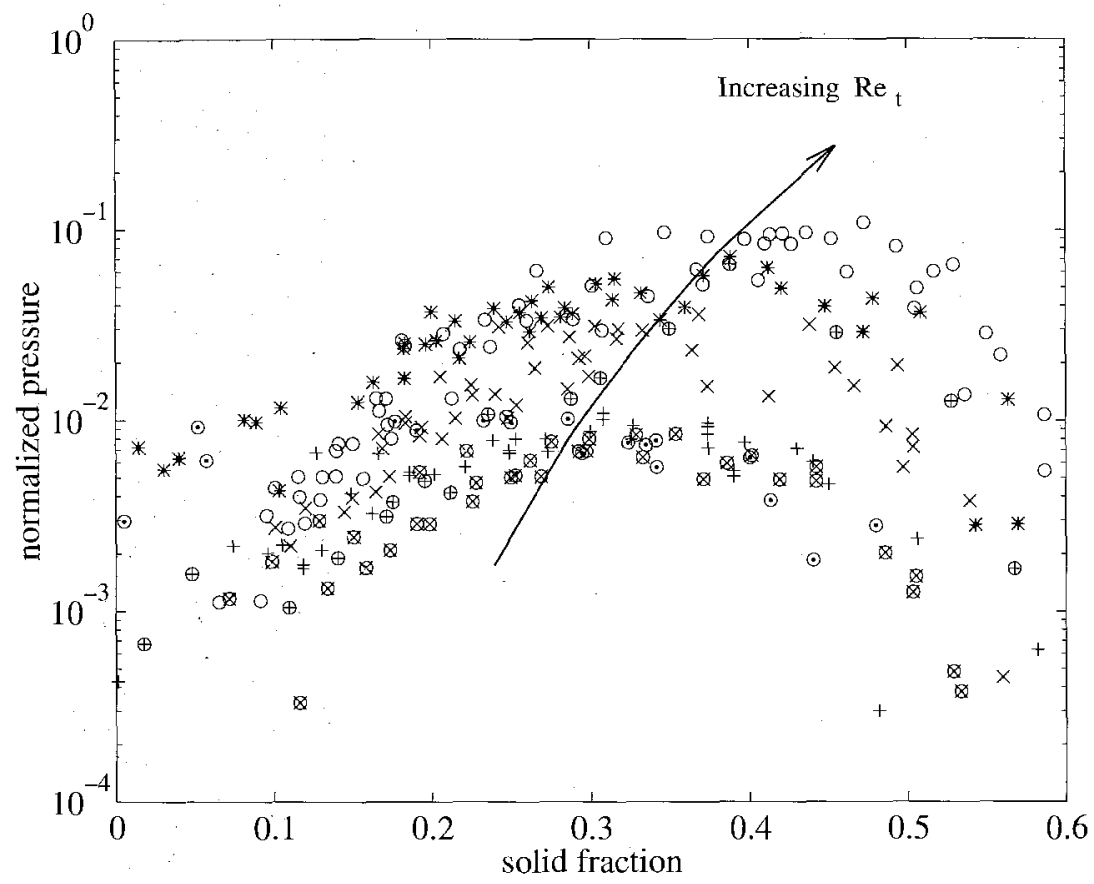

Figure 2. Normalized particle pressure as a function of the solid fraction, $v$. Pressures normalized by $\left(\rho_{p} u_{t}^{2}\right) / 2$. Results for all particles and tests sections in a fluidized bed. For symbol identification, see Table I.

where $P(t)$ is the measured pressure pulse signal and $\tau$ is the duration of the pulse.

The calibration of the transducer provided by the manufacturer was corroborated by measuring the pressure pulses produced by particles impacting at known velocities in air, and by comparing with the predictions from the Hertzian theory of contact [12].

The granular pressures measured in a fluidized bed by Zenit et al. [14] are shown in Figure 2 as a function of the solid fraction. The particle pressure is nondimensionalized by $\left(\rho_{p} u_{t}^{2}\right) / 2$. The same general behavior was found for all the particles tested. At low concentrations the particle pressure is small. In this regime the particles are free to move and collide occasionally; therefore few particles collide with the transducer per unit time, making the event rate small. On the other hand, at high solid fractions, collisions are more likely to occur and these collisions occur at small velocities producing low impulse collisions, which result in a low: value of the particle pressure. At intermediate concentrations (from 30 to 35\%) the particle pressure reaches a maximum.

The presentation of the normalized particle pressure in Figure 2 is consistent with the dimensionless representation used in the original work by Batchelor [2]. However it is clear that the results are also a function of other parameters such 


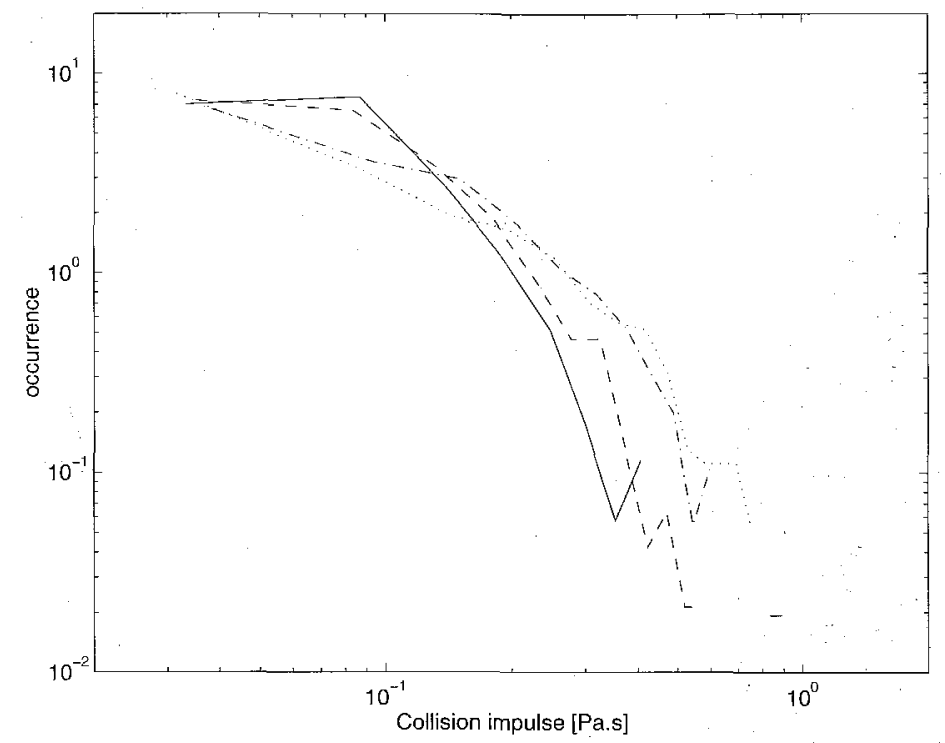

Figure 3. Probability density function of collision impulse. Fluidized bed with $3 \mathrm{~mm}$ glass particles in a $10.2 \mathrm{~cm}$ test section. $(-) v=0.503,(-) v=0.438,(-\cdot) v=0.318$, $(\cdots) v=0.194$

as the Reynolds number, $\operatorname{Re}_{t}$, or the density ratio, $\rho_{p} / \rho_{f}$. In Figure 2, the larger Reynolds numbers occur at the top of the band of data and the smaller values at the bottom. One exception to this is the data for steel particles which also happens to have the largest density ratio.

\subsection{PROBABILITY DENSITY FUNCTIONS}

Zenit et al. also performed detailed statistical analysis in which probability density functions (PDF) were generated both for the collision impulse and the collision duration. Figures 3 and 4 show typical normalized PDFs obtained for $3 \mathrm{~mm}$ glass particles in the $10.2 \mathrm{~cm}$ test section. Figure 3 shows how the distribution of collision impulse changes with solid fraction. Clearly, in concentrated beds, collisions of low impulse are predominant. As the solid fraction decreases the distribution becomes wider, indicating an increase in the occurrence of higher impulse collisions.

More unexpected was the form of the PDF for the collision duration, shown in Figure 4. For all the solid fractions tested the duration exhibits a distribution with two distinct peaks. The first peak (short duration pulses) occurs at approximately $15 \mu \mathrm{s}$ and its magnitude appears to increase with decreasing solid fraction. The second peak (long duration pulses) occurs at approximately $37 \mu$ s and becomes narrower for lower solid fractions. 


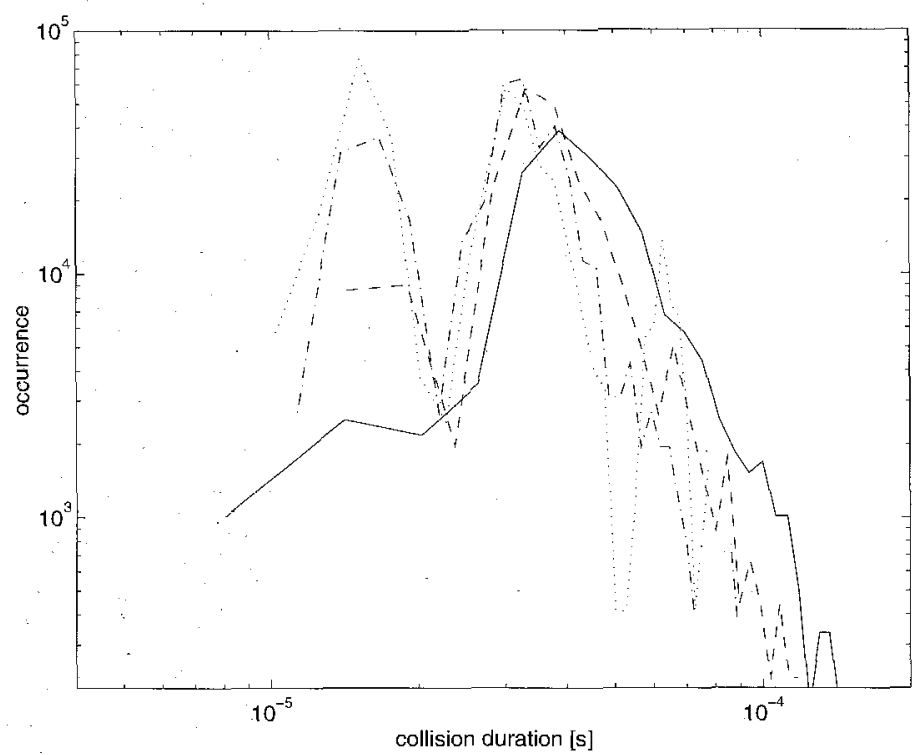

Figure 4. Probability density function of collision duration. Fluidized bed with $3 \mathrm{~mm}$ glass particles in a $10.2 \mathrm{~cm}$ test section. (-) $v=0.503,(-) v=0.438,(--) v=0.318$, $(\cdots) v=0.194$.

The long duration pulses have impulses and strengths that scale according to predictions from Hertzian theory [8] for a particle impacting a solid surface. On the other hand, the short duration pulses are always associated with low impulse collisions.

To help understand the two pulse types, the visualization system shown in Figure 5 was devised. An observation window was installed near the pressure transducer and a high speed digital camera, able to record images up to 500 frames per second, was positioned to observe the face of the transducer. The camera was synchronized with the data acquisition system so that the transducer could be observed simultaneously with the signal registered.

The long duration pulses were confirmed to be the result of direct collisions of particles with the transducer. Particles were observed to collide with the face of the transducer at many different speeds and incident angles. The strength and duration of these collisions were confirmed to be in accordance with Hertzian predictions.

The short duration events were found to be produced, not by actual contacts between particles and the transducer, but rather by collisions between particles in the bulk that occur in the vicinity of the pressure transducer. The pressure pulse produced by such a collision travels from the point of occurrence to the transducer through the interstitial fluid. The intensity of these pulses appeared to be related to the impact velocity of the collision, the orientation of the colliding particles with respect to the transducer and the distance from the collision to the transducer. The 


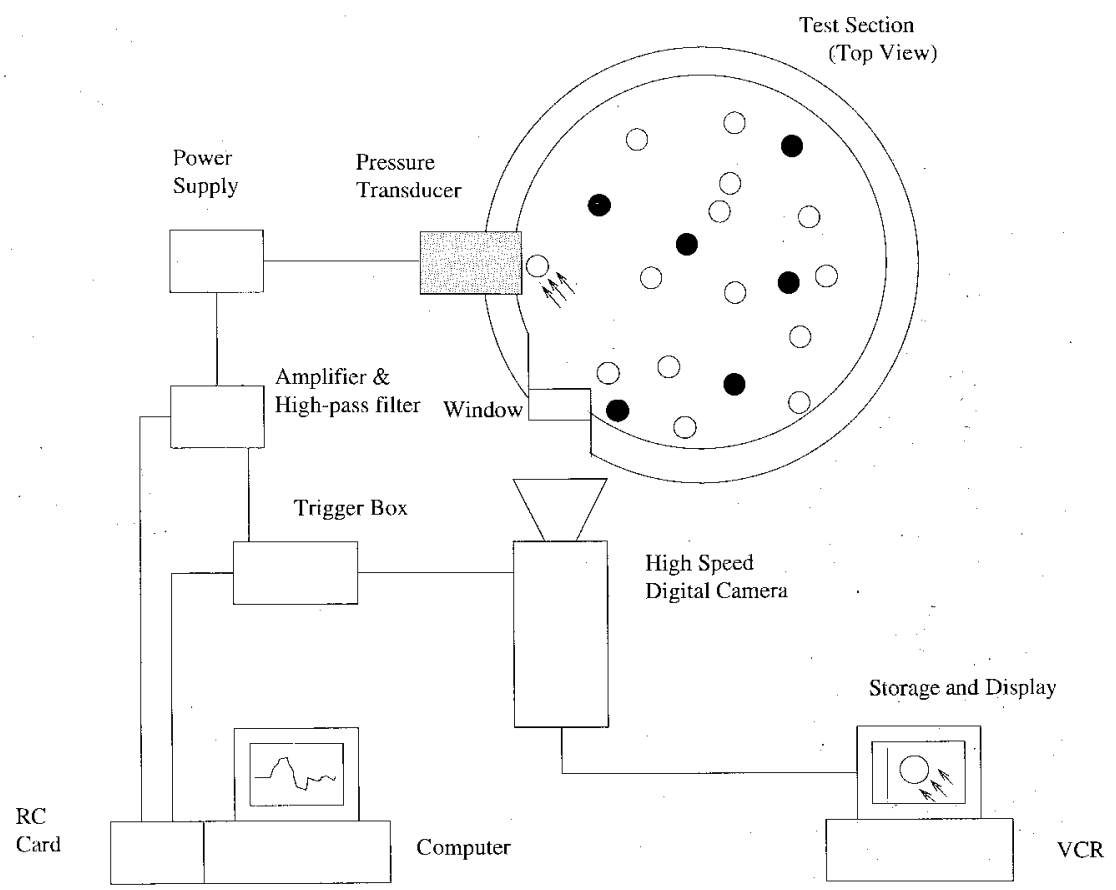

Figure 5. Schematic of experimental setup used to obtain the images of the moving particles and pressure pulses simultaneously.

duration of these events is in accordance with Hertzian theory for particle-particle collisions.

Clearly, then, there are two distinct contributions to the particle pressure. First, there is a direct contribution resulting from direct collisions of particles with the pressure transducer. Second, there is a radiated contribution resulting from collisions between particles in the bulk. These impulsive pressures are radiated through the liquid. In the following sections investigations are presented that are aimed to further understand these two contributions.

Although the occurrence of the two different pressure pulses could be equally frequent, their net contribution to the total particle pressure is significantly different, because the magnitude of the direct component impulses is, in general, larger than the magnitude of the radiated component.

\subsection{DiRECT CONTRIBUTION}

The direct component of the particle pressure is generated by collisions of particles with the surface of the transducer. In general, the magnitude and duration of a collision depends on the elastic properties of the colliding surfaces, the impact velocity, the mass of the particle and the fluid properties. Dry collisions of particles 
Type I

Direct collision.
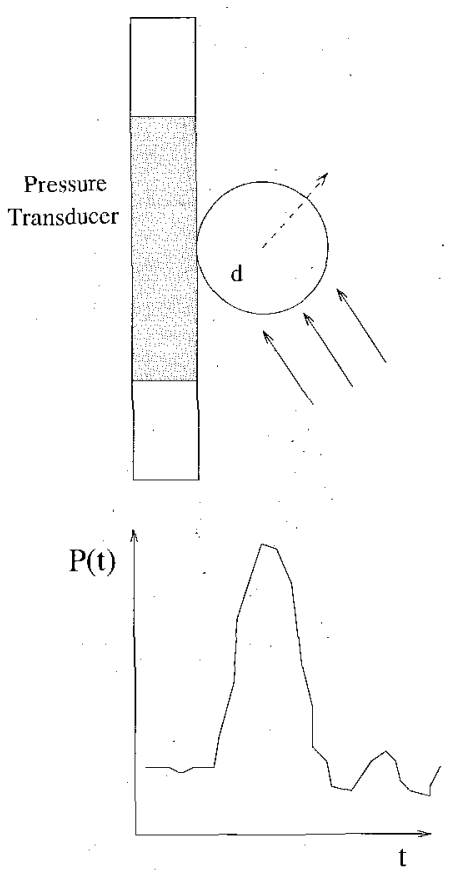

Type II

Collision near the transducer.
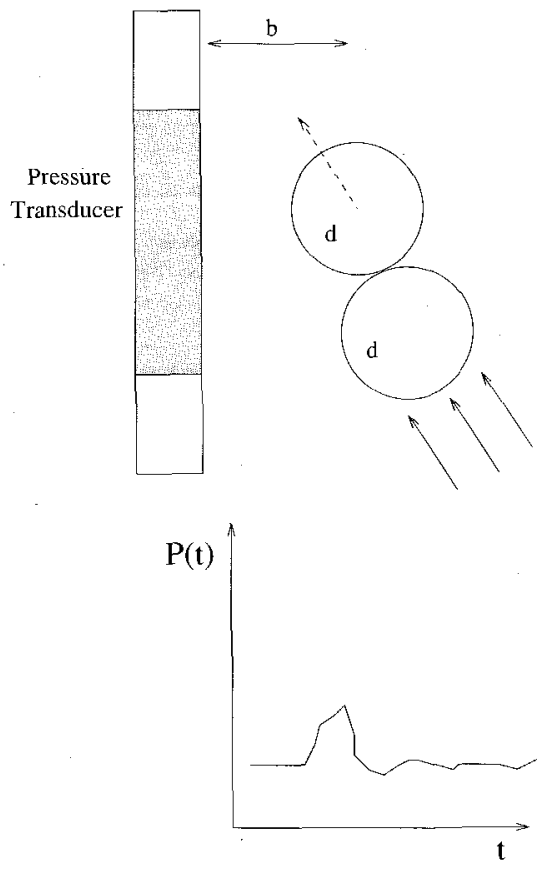

Figure 6. Types of pressure pulses that contribute to the particle pressure. Sketch of the collisions and type of pressure pulse recorded. The solid arrows represent the motion before contact, the dashed arrows the motion after contact.

have been extensively studied in the past [5, 8]. In a dry collision the surrounding fluid plays little or no role in the mechanics of contact and rebound.

Wet collisions are less understood. Before actual contact between the solid surfaces occurs, large hydrodynamic pressures are generated in the gap between the surfaces. Several researchers have studied the mechanics of collisions when the effects of the surrounding fluid are not negligible. Davis et al. [3] and Kytömaa and Schmid [10] focused on small Reynolds numbers, at which the moving particle is predicted to come to a complete stop before contacting the wall. Therefore rebound does not occur. A basic understanding of collisions at larger Reynolds numbers has yet to be developed. Only a limited number of experimental studies have focused on this topic [11,13].

In the present investigation, measurements were made of the pressure impulse generated by the collision of a particle with the flush mounted transducer. A particle was suspended by a fine string and swung pendulum-like to produce controlled and repeatable collisions. The particle was released from rest at some initial angle to produce various incident velocities. 


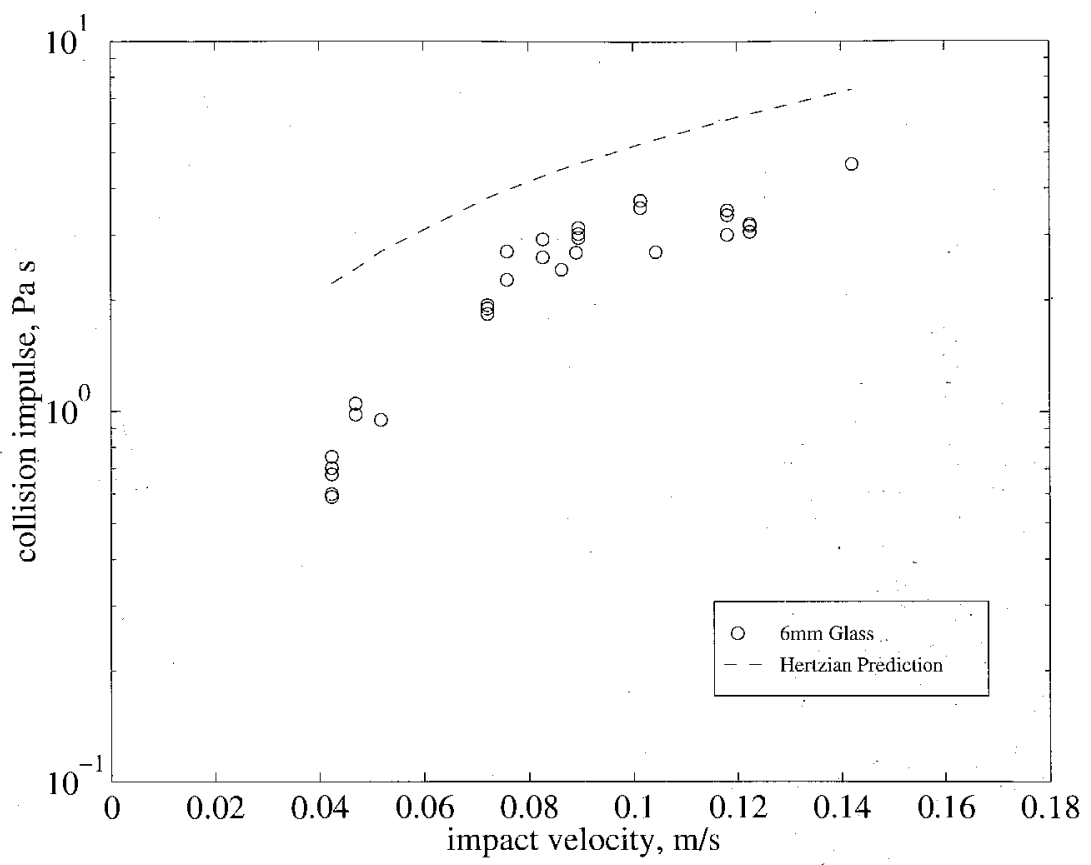

Figure 7. Immersed normal collision of a particle against a flat wall. Pressure impulse as a function of predicted maximum impact velocity for a $6 \mathrm{~mm}$ glass particle immersed in water.

Typical measured impulse pressures are plotted as a function of impact velocity in Figure 7. The velocity plotted horizontally is the predicted maximum velocity calculated from a damped pendulum equation; it is a function of the initial position of the particle. The prediction from Hertzian theory is also shown in Figure 7. This prediction neglects the effects of the interstitial fluid and therefore represents an upper limit on the impulse. The difference between the measured pulses and the prediction therefore results from interstitial fluid effects; note that it appears to be larger for smaller impact velocities. Thus for smaller approach velocities, the viscous effects become more important $[11,13]$.

\subsection{RADIATED CONTRIBUTION}

The radiated component of the particle pressure is generated by particle-particle collisions that occur in the bulk of the flow. The impulsive change of velocity resulting from a collision generates a pressure pulse that is transmitted through the liquid. The strength of the measured pulse is related to the impact velocity, the orientation of the colliding particles with respect to the transducer and the distance from the collision to the transducer. 


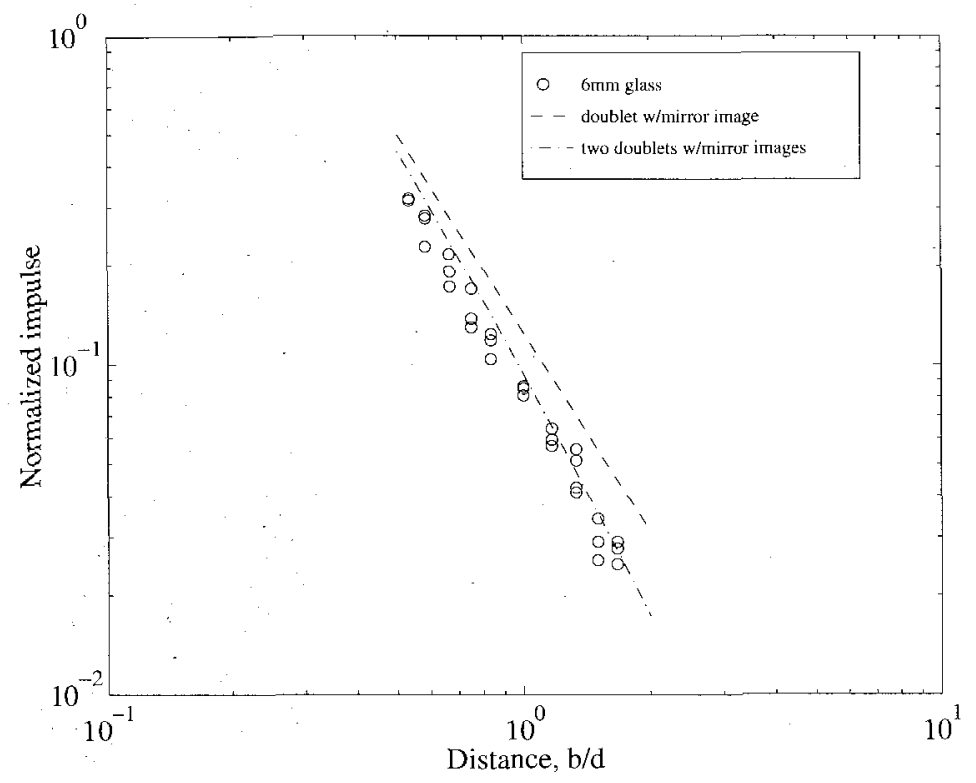

Figure 8. Measured impulse as a function of distance from the wall for a $6 \mathrm{~mm}$ glass particle colliding with a similar particle in water. Velocity of the target particle after impact $=0.075 \mathrm{~m} / \mathrm{s}$. The lines refer to the theoretical expressions described in the text

The existence of this radiated component of the collisional particle pressure has not been previously recognized. In the present study, it was further investigated in the following way. The pendulum experiment described in Section 2.2 was modified to generate binary collisions of particles. A second 'target' particle was suspended at rest at a distance from the transducer surface. The 'impact' particle was released starting from rest at some initial angle, as in the direct collision experiment. When the two particles collide, the impulsive accelerations produce a pressure pulse, which is transmitted through the fluid and detected by the pressure transducer. The velocities of the target and impact particles after the collision occurred were measured using a high-speed digital camera.

A typical result for a pair of $6 \mathrm{~mm}$ glass particles in water is shown in Figure 8. The plot shows the radiated impulse generated for different distances between the target particle and the transducer (for a fixed impact velocity). It can be observed that the strength of the measured pressure pulse decreases as the distance from the transducer increases. The magnitude of the impulses is much smaller than those in the direct collision experiments. Tests were also performed for different impact velocities, as well as for different particle sizes and densities [12]. The impulses shown in Figure 8 are normalized by $\rho_{f} U d$.

To further investigate the nature of these pressure pulses, an analysis was carried out using the pressure-impulse theory [1]. Neglecting both convective inertial and viscous effects, the equations of motions of the fluid reduce to 


$$
\frac{\partial \mathbf{u}}{\partial t}=-\frac{1}{\rho_{f}} \nabla P .
$$

This equation can be time-integrated through the impact time duration from $t=0$ to $t=\tau$ to obtain

$$
\mathbf{u}_{\tau}-\mathbf{u}_{0}=-\frac{1}{\rho_{f}} \nabla I,
$$

where $\mathbf{u}_{0}$ and $\mathbf{u}_{\tau}$ are the particle velocities before and after the collision and $I$ is the pressure impulse (as defined in Equation (2)). If the flow is incompressible, then it follows that

$$
\nabla^{2} I=0 \text {. }
$$

Thus, the impulse generated by the impulsive motion of a body is very similar to the velocity potential for the steady potential flow around that same body. For example the impulse, $I$, produced by a sphere, of diameter $d$, given a sudden change $U$ in its velocity in the $x$-direction, will be given by a doublet so that

$$
I=\frac{1}{2} \rho_{f} U\left(\frac{d}{2}\right)^{3} \frac{x+b}{\left((x+b)^{2}+y^{2}\right)^{3 / 2}},
$$

where the center of the sphere is at $x=-b, y=0$.

To obtain an approximate solution when there is a solid wall at $x=0$, an image doublet is placed at $x=+b, y=0$ in order to satisfy the condition of zero velocity normal to the wall. Then

$$
I=\frac{1}{2} \rho_{f} U\left(\frac{d}{2}\right)^{3}\left(\frac{x+b}{\left((x+b)^{2}+y^{2}\right)^{3 / 2}}-\frac{x-b}{\left((x-b)^{2}+y^{2}\right)^{3 / 2}}\right)
$$

and this would result in a measured impulse at the origin (the face of the transducer) given by

$$
I=\rho_{f} U\left(\frac{d}{2}\right)^{3}\left(\frac{1}{b^{2}}\right)
$$

This is plotted as the dashed line in Figure 8. The prediction slightly overestimates the experimental measurements but follows the trend quite well.

To generate a more accurate prediction, it is noted that both the target and impact particles produce an impulse. This can be simulated by placing one doublet of positive strength, $\rho_{f} U$, at $x=-b$ and a second one with negative strength, $-\rho_{f} U$, at $x=-(b+d)$. In addition, image doublets are placed at $x=b$ and $x=b+d$. This leads to

$$
\begin{aligned}
I= & \frac{1}{2} \rho_{f} U\left(\frac{d}{2}\right)^{3}\left(\frac{x+b}{\left((x+b)^{2}+y^{2}\right)^{3 / 2}}-\frac{x+b+d}{\left((x+b+d)^{2}+y^{2}\right)^{3 / 2}}\right. \\
& \left.-\frac{x-b}{\left((x-b)^{2}+y^{2}\right)^{3 / 2}}+\frac{x-b-d}{\left((x-b-d)^{2}+y^{2}\right)^{3 / 2}}\right)
\end{aligned}
$$


and an approximate impulse at the transducer given by

$$
I=\rho_{f} U\left(\frac{d}{2}\right)^{3}\left(\frac{2 d b+d^{2}}{\left.b^{2}(b+d)^{2}\right)}\right) .
$$

Of course, higher order singularities could be added for greater numerical accuracy. However, the construction above should represent the essence of the mechanics of the radiated pulse caused by the collision of two particles near a boundary.

The dashed-dotted line in Figure 8 is calculated from Equation (10), and agrees very well with the experimental measurements. The same kind of agreement between the experiments and the model was obtained for most of the particles tested, with the exception of particle whose terminal Reynolds number was small [12].

\section{Conclusions}

This paper presents an overview of several investigations (both experimental and analytical) examining the two contributions to the granular pressure in a liquid fluidized bed observed by Zenit et al. [14]. In that earlier paper the particle pressure generated by collisions of particles in liquid-solid flows was measured using a highfrequency response pressure transducer. The measured pressure was found to be small for dilute mixtures and to increase with increasing concentration. It reached a maximum at an intermediate concentrations, and then decreased as the mixture approached close packing. The magnitude of the measured pressure was found to scale with the density of the particles, $\rho_{f}$, and the particle terminal velocity, $u_{t}$. However, other parameters (such as the Reynolds number) influence the particle pressure. The analysis of the PDFs of collision duration combined with detailed observations, led to the discovery of two distinct contributions to the collisional particle pressure.

The two contributions (or types of pulses) were investigated experimentally. By means of a pendulum experiment, controlled collisions were generated. To study the direct component, collisions of particles with the pressure transducer were observed. The impulse was quantified and plotted as a function of the impact velocity. Results were compared with predictions from Hertzian theory of contact. The difference between the measurements and the prediction were attributed to the effects of the interstitial fluid. To investigate the radiated component, binary collisions were generated by adding a second suspended particle positioned at a particular distance from the wall. The pressure front that resulted from the collision was measured by the pressure transducer. The magnitude of the radiated impulse was found to be related to the impact velocity, the diameter of the particles and the distance from the wall. Following a impulse-pressure analysis, a simplified model was proposed. The predictions of the model compared well with the experimental measurements. 


\section{Acknowledgement}

The National Council for Science and Technology of Mexico (CONACYT) is acknowledged for partially supporting $\mathrm{R}$. Zenit during his graduate sojourn at the California Institute of Technology.

\section{References}

1. Batchelor, G.K., An Introduction to Fluid Dynamics. Cambridge University Press, Cambridge, UK (1967).

2. Batchelor, G.K., 'A new theory of the instability of a uniform fluidized bed. J. Fluid Mech. $\mathbf{1 9 3}$ (1988) $75-110$.

3. Davis, R.H., Serayssol, J.M. and Hinch, E.J., The elastohydrodynamic collision of two spheres. J. Fluid Mech. 163 (1986) 479-497.

4. Foscolo, P.U. and Gibilaro, L.G., Fluid dynamic stability of fluidized suspensions. Chem. Eng. Sci. 39 (1987) 1485-1500.

5. Goldsmith, W., Impact, The Theory and Physical Behavior of Colliding Solids. E. Arnold Publishers, London (1960).

6. Jackson, R., The mechanics of fluidized beds. Part I: The stability of the state of uniform fluidization. Trans. Inst. Chem. Eng. 41 (1963) 13-4l.

7. Jackson, R., Hydrodynamic stability of fluid-particle systems. In: Davidson, J., Clift, R. and Harrison, D. (eds), Fluidization. Academic Press, London, second edition (1985).

8. Johnson, J.K., Contact Mechanics. Cambridge University Press, Cambridge, U.K. (1987).

9. Kytömaa, H.K. and Brennen, C.E., Some observations of flow patterns and statistical properties of three component flows. J. Fluids. Eng. 110 (1986) 76-84.

10. Kytömaa, H.K. and Schmid, P.J., On the collision of rigid spheres in a weakly compressible fluid. Phys. Fluids A 4(12) (1992) 2683-2689.

11. McLaughlin, M.H., An experimental study of particle-wall collision relating of flow of solid particles in a fluid. Engineer Degree Thesis. California Institute of Technology, Pasadena, CA (1968).

12. Zenit, R., Collisional mechanics in liquid-solid flows. PhD Thesis, California Institute of Technology, Pasadena, CA (1997).

13. Zenit, R. and Hunt, M.L., Influence of fluid properties on submerged collision of particles. In: Roco, M.C. (ed.), ASME International Symposium of Liquid-Solid Flows, Vancouver, Canada (1997).

14. Zenit, R., Hunt, M.L. and Brennen, C.E., Collisional particle pressure measurements in solidliquid flows. J. Fluid Mech. 353 (1997) 26l-283. 